\title{
Kakayahang lingguwistik at diskorsal ng baitang 10 - special program on journalism: Isang pagsusuri
}

\author{
Pante, Diana Joy B. \\ Bogña Integrated School, Philippines (dianajoypante17@gmail.com)
}

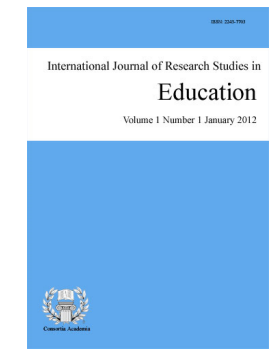

Accepted: 23 January 2022

ISSN: 2243-7703 Online ISSN: 2243-7711

OPEN ACCESS

\section{Abstract}

The challenge and impact of the pandemic on education is greatly affected around the world. Closing of schools has become one of problem for everyone. However, due to the desire to continue the education, a modular method was used in different schools. This research aimed to determine the level of linguistic and discoursal skills of Grade 10 students in the Special Program on Journalism (Radio Broadcasting) of Bogña Integrated School. Identify the effects of Modular Distance Learning on students' ability, problems faced by students in Modular Distance Learning and suggest activities to further develop students' linguistic and discourse skills. Quantitative-Qualitative and descriptive analysis design in data collection. Purposive sampling was used to identify respondents. Consists of 22 respondents from Grade 10Special Program on Journalism (Radio Broadcasting) and 4 teachers. Frequency count, mean, percentage and ranking were used in the analysis and interpretation of the data. In total the level of linguistic skills of the students were $82 \%$ which means at the moderate level of proficiency. In total the level of discoursal skills of the students were $83 \%$ which is at the moderate skill level. significant impact on students 'linguistic and discursive ability. These effects caused them to not be able to demonstrate their skill. In conclusion the study was able to identify the existing challenges to the ability of the participants. The result of this study can serve as an instrument for the improvement of existing school programs such as imparting knowledge to the student using modular method.

Keywords: linguistic, discoursal, effect, journalism, modular distance learning 


\section{Kakayahang lingguwistik at diskorsal ng baitang 10 - special program on journalism: Isang pagsusuri}

\section{Introduksyon}

Mahalaga ang gampanin ng edukasyon sa paghubog ng kakayahan at kasanayan ng bawat mag-aaral. Ito rin ang nagsisilbing pundasyon upang malinang ang kanilang natatagong kaalaman at talento sa iba't ibang larang. Ito rin ang nagsisilbing gabay sa pagkamit ng tagumpay sa larangan ng pag-aaral at pagkatuto. Sa panahon ng mabilis na pagkalat ng Covid-19 sa buong mundo malaking suliranin ito sa larangan ng edukasyon sa paghahatid ng pagkatuto sa mga mag-aaral. Maraming mga paaralan din ang nagsara dahil sa malaking banta sa kalusugan ng mga guro, magulang at mag-aaral ang pagkalat ng virus. Malaki din ang epekto nito sa kasanayan ng bawat mag-aaral.

Ang Kagawaran ng Edukasyon sa Amerika, 2020) ay mabilisang nilinaw na sa panahon ng pandemya, ang bawat paaralan ay dapat pa ring ipagpatuloy ang pagtuturo gamit ang iba't ibang pamamaraan ng pagtuturo,ngunit habang ang mag-aaral ay nagtatamo na makamit ang kanilang pangangailangan, ang mga hadlang sa pagtamo ng pagkatuto ay kailangang malimitahan. Ayon kay Llego (2020), ang "Distance learning Modalities" ay maaaring makatulong sa pagpapa-unlad ng access sa edukasyon, at paglinang sa pagkatuto, maging sa pagpapalaganap ng "Basic-Education- Learning Continuity Plan" sa pagtugon sa hamon ng pandemya tulad ng ng Covid-19, pagkalat ng nakahahawang sakit tulad ng Ebola, at mga natural na kalamidad. Sa pamamagitan ng pagsunod sa apat na pamamaraan ng "distance learning modalities" maaaring maging susi ito upang maitawid ang pagtuturo at pagkatuto.

Ayon kay Badayos (2008), ang pagsasalita ang pinakamagiting sa pagpapahayag ng wika. Halos lahat ng bata ay may taglay nang katatasan sa pagsasalita bago pa man sila pumasok sa paaralan. Dahil sa ganitong kalagayan, inaakala ng maraming guro na hindi na dapat bigyan-pansin ang paglinang ng kasanayang ito sa mga paaralan lalo na sa panahon ng pandemya. Ngunit may mga pag-aaral na nagsasabing malaki ang kapakinabangang matatamo ng mga mag-aaral sa kanilang mga pakikilahok sa mga gawain sa pagsasalita maging ito man ay pormal o di-pormal. Makatutulong ito upang malinang ang kakayahang diskorsal ng mag-aaral. Sa pananaw ng lingguwistang si Chomsky (1965), ang kakayahang lingguwistiko ay isang ideyal na sistema ng di-malay o likas na kaalaman ng tao hinggil sa gramatika na nagbibigay sa kaniya ng kapasidad na gumamit at makaunawa ng wika. Pumapaloob dito ang kaalaman ng tao na pag-ugnayin ang tunog o mga tunog at kahulugan nito. lba ito sa isinasaad ng lingguwistikong pagtatanghal (linguistic performance) o ang aplikasyon ng sistema ng kaalaman sa pagsusulat o pagsasalita.

Sa DepEd Order No. 12, s. 2020, ang integrasyon ng awtput sa "Basic Education- Learning Continuity Plan”, ang tanggapan ng Kalihim ay nagpalabas ng sumusunod na prinsipyo: una, protekahan ang kalusugan at kaligtasan ng bawat mag-aaral at lahat ng kawani ng pamahalaan at pagsugpo sa pagkalat ng Covid-19. Ikalawa, sikapin ang pagpapalaganap ng sulong edukalidad ng K-12 Kurikulum, katugmaan ng mga kagamitang panturo ayon sa mga mahahalagang kasanayang pampagkatuto, pagsasanay ng mga guro at mga mag-aaral at oryentasyon sa mga magulang o tagapangalaga ng bawat mag-aaral. Batay sa Konstitusyon ng Republika ng Pilipinas, 1987 sa Artikulo XIV Seksyon Seksyon 1, dapat pangalagaan at itaguyod ng estado ang Karapatan ng lahat ng mga mamamayan sa mahusay na edukasyon sa lahat ng antas. Dapat magsagawa ng angkop na mga hakbang upang matamo ng lahat ang gayong edukasyon. Ang nabanggit na batas ay mababasa rin sa Republic Act (RA) No. 10533 o ang Enhanced Basic Education Act of 2013.

Sa Broadcast Code of the Philippines (2007) as amended 2011. Hinihikayat ang lahat ng magbabalita sa radyo na ang balitang isasahimpapawid ay may katotohanan, walang kinikilingan, at napapanahon. Sa isang modelo ng pagtataya ng pagkatuto na binuo ni Cabigao (2021), ngayong Covid-19 pandemic, binigyang-diin niya 
ang tatluhang-ugnayan ng mga salik na nakaaapekto sa pagtataya. Binanggit niya na upang maging epektibo sa pagtataya, mahalaga ang ugnayan ng guro at mag-aaral. Malaking hamon sa panahon ng pandemyang ito ang pagtataya ng pagkatuto ng mga mag-aaral.

Batay sa naging obserbasyon at pakikipanayam na mananaliksik sa mga guro at mag-aaral malaki ang suliranin kinakaharap sa modular distance learning sa paghahatid ng pagkatuto sa mag-aaral. Kung kaya't layunin ng pag-aaral na matukoy ang lebel ng kakayahang lingguwistik at diskorsal ng mga mag-aaral sa Special Program on Journalism. Nais ding bigyang tugon ng mananaliksik ang mga hamong kinahaharap ng mga mag-aaral sa paglinang ng kakayahang lingguwistik at diskorsal. Gayundin matulungan ang mga guro sa paglinang ng kakayahan ng mga mag-aaral.

\section{Paglalahad ng Suliranin}

Layunin ng pag-aaral na ito masuri ang kakayahang lingguwistik at diskorsal ng mga mag-aaral sa asignaturang Journalism ng Baitang 10- Special Special Program on Journalism (Radio Broadcasting) ng Bogña Integrated School (BIS) sa Taong Panuruan 2021-2022. Matukoy ang epekto ng Modular Distance Learning sa kakayahan ng mga mag-aaral, suliraning kinakaharap ng mga mag-aaral sa Modular Distance Learning at makapagbigay mungkahing gawain ang maaaring gawin upang mas higit na malinang ang kakayahang lingguwistik at diskorsal ng mga mag-aaral sa Baitang 10- Special Program on Journalism (Radio Broadcasting) ng BIS.

\section{Pamamaraang Ginamit}

Natiyak sa pag-aaral na ito kakayahang lingguwistik at diskorsal ng mga mag-aaral sa asignaturang journalism ng Baitang 10- Special Special Program on Journalism (Radio Broadcasting) ng Bogña Integrated School sa Taong Panuruan 2021-2022. Kwantitatibo-Kwalitatibong at deskriptib analisis ang disenyo sa paglikom ng datos. Purposive sampling ang ginamit sa pagtukoy ng mga kalahok. Binubuo ng 22 kalahok mula sa Baitang 10- Special Program on Journalism (Radio Broadcasting) at 4 na guro. Ginamit ang mga tala ng resulta sa mga gawaing pagkatuto "Learning Activity Sheet" at pagganap o performans at open-ended na tanong para sa panayam sa pamamagitan ng via messenger/ email mula sa mga guro. Inanalisa, sinuri, at binigyan ng interpretasyon sa tulong ng nararapat na estadistika tulad ng frequency count, mean, bahagdan at pagrarango.

\section{Mga Natuklasan}

Inilahad dito ang pagsusuri at interpretasyon sa mga nakalap na datos ukol sa ang kakayahang lingguwistik at diskorsal ng mga mag-aaral sa asignaturang Journalism ng Baitang 10- Special Special Program on Journalism (Radio Broadcasting) ng Bogña Integrated School sa Taong Panuruan 2021-2022. Makikita sa Talahanayan 1A ang lebel ng kakayahang ligguwistik ng mga mag- aaral sa asignaturang journalism ng Baitang 10- sa Special Program on Journalism (Radio Broadcasting). Napatunayan na 6 sa mag-aaral ang nakakuha ng 90-100\%, wala namang nakakuha ng 85-89\%, sinundan ito ng 5 mag-aaral na nakakuha ng 80-84\%, samantala 11 naman ang nakakuha ng 75-79\%. Sa kabuuan ang lebel ng kakayahang lingguwistik ng mga mag-aaral ay $82 \%$ na nangangahulugang nasa katamtamang lebel ng kasanayan. Kung kaya’t nangangailangan pa rin ng puspusang gabay ang mga mag-aaral upang mas mapaangat pa ang kanilang performans. Kailangan din ang pokus upang mas lalo pang malinang ang kanilang kakayahan.

Matutunghayan naman sa Talahanayan 1B lebel ng kakayahang diskorsal ng mga mag- aaral sa asignaturang journalism ng Baitang 10- sa Special Program on Journalism (Radio Broadcasting). Ipinapakita sa talahanayang ito ang naging resulta ng pag-aaral na 3 sa mag-aaral ang nakakuha ng 90-100\%, 8 naman ang nakakuha ng 85-89\%, sinundan ito ng 5 mag-aaral na nakakuha ng 80-84\%, 11 naman ang nakakuha ng 75-79\%, Sa kabuuan ang lebel ng kakayahang diskorsal ng mga mag-aaral ay $83 \%$ na nasa katamtamang lebel ng kasanayan. Kung kaya't nangangailangan pa rin ng puspusang gabay ang mga mag-aaral upang mas mapaangat pa ang kanilang performans lalo na ang kasanayan sa pagsasalita. Kailangan din ang pokus upang mas lalo pang malinang ang 
Pante, D. J. B.

kanilang kakayahan.

\section{Talahanayan 1A}

Lebel ng Kakayahang Ligguwistikng mga Mag-aaral

\begin{tabular}{cccl}
\hline Marka & frequency & Bahagdan $(\%)$ & \\
\hline $90-100$ & 6 & 27 & Napakataas \\
$85-89$ & 0 & 0 & Mataas \\
$80-84$ & 5 & 23 & Katamtaman \\
$75-79$ & 11 & 50 & Mababa \\
Mababa sa 75 & 0 & 0 & Hindi nakamit ang kasanayan \\
Kabuoan & & 100 & PL-82\% Katamtaman \\
\hline D.O. No. 8, s. 2015 (Batayan sa Pagkuha ng Performans Lebel) &
\end{tabular}

D.O. No. 8, s. 2015 (Batayan sa Pagkuha ng Performans Lebel)

\section{Talahanayan 1B}

Lebel ng Kakayahang Diskorsal ng mga Mag-aaral

\begin{tabular}{cccl}
\hline Marka & frequency & Bahagdan $(\%)$ & Deskripsyon \\
\hline $90-100$ & 3 & 14 & Napakataas \\
$85-89$ & 8 & 0 & Mataas \\
$80-84$ & 5 & 36 & Katamtaman \\
$75-79$ & 11 & 50 & Mababa \\
Mababa sa 75 & 0 & 0 & Hindi nakamit ang kasanayan \\
Kabuoan & & 100 & PL-83\% Katamtaman \\
\hline
\end{tabular}

D.O. No. 8, s. 2015 (Batayan sa Pagkuha ng Performans Lebel)

Sa isang modelo ng pagtataya ng pagkatuto na binuo ni Cabigao (2021), ngayong Covid-19 pandemic, binigyang-diin niya ang tatluhang-ugnayan ng mga salik na nakaaapekto sa pagtataya. Binanggit niya na upang maging epektibo sa pagtataya, mahalaga ang ugnayan ng. Malaking hamon sa panahon ng pandemyang ito ang pagtataya ng pagkatuto ng mga mag-aaral. Sa pananaw ng lingguwistang si Chomsky (1965), ang kakayahang lingguwistiko ay isang ideyal na sistema ng di-malay o likas na kaalaman ng tao hinggil sa gramatika na nagbibigay sa kaniya ng kapasidad na gumamit at makaunawa ng wika. Pumapaloob dito ang kaalaman ng tao na pag-ugnayin ang tunog o mga tunog at kahulugan nito. lba ito sa isinasaad ng lingguwistikong pagtatanghal (linguistic performance) o ang aplikasyon ng sistema ng kaalaman sa pagsusulat o pagsasalita.

Makikita sa Talahanayan 2A epekto ng modular distance learning sa kakayahang lingguwistik ng mga mag-aaral sa Baitang 10- sa asignaturang Special Program on Journalism (Radio Broadcasting) na kung saan 17 may kahirapan sa paggamit ng wastong bantas, gamit ng malaki at maliit na titik at baybay sa pagkakabuo ng mga balita. Sinundan naman 15 hindi lohikal na nakaayos ang mga ideya sa pagsulat ng balita. Nabanggit din na 12 na walang kaangkupan sa pagpili ng mga salita sa pagbuo ng balita, hindi komprehensibo ang pagkakabuo sa mga ideya sa pagkakabuo ng balita at walang malinaw na pagkakalagay ng mga impormasyon sa pagkakabuo ng balita. Samantala 10 nahihirapan sa pagpili ng angkop ang paraan ng pagkakasulat ng balita. Nagbigay naman pahayag na 7 walang batayan ang mga impormasyong isinusulat sa balita.

\section{Talahanayan 2A}

Epekto ng Modular Distance Learning sa Kakayahang Lingguwistik

\begin{tabular}{lcc}
\hline \multicolumn{1}{c}{ Epekto ng Modular Distance Learning sa Kakayahang Lingguwistik } & f & Ranggo \\
\hline Walang kaangkupan sa pagpili ng mga salita sa pagbuo ng balita & 12 & 4 \\
Hindi komprehensibo ang pagkakabuo sa mga ideya sa pagkakabuo ng balita & 12 & 4 \\
May kahirapan sa paggamit ng wastong bantas, gamit ng malaki at maliit na titik at baybay sa & 17 & 1 \\
pagkakabuo ng mga balita & 12 & 4 \\
Walang malinaw na pagkakalagay ng mga impormasyon sa pagkakabuo ng balita & 8 & 7 \\
Walang batayan ang mga impormasyong isinusulat sa balita & 10 & 6 \\
Nahihirapan sa pagpili ng angkop ang paraan ng pagkakasulat ng balita. & 15 & 2 \\
Hindi lohikal na nakaayos ang mga ideya sa pagsulat ng balita & & \\
\hline
\end{tabular}

Batay sa resulta ng pag-aaral sa Talahanayan 2B Epekto ng Modular Distance Learning sa Kakayahang Diskorsal ng mga Mag-aaral sa Baitang 10- sa asignaturang Special Program on Journalism (Radio 
Kakayahang lingguwistik at diskorsal ng baitang 10 - special program on journalism: Isang pagsusuri

Broadcasting). Nahihirapan sa paglalapat nang angkop na lakas ng boses o tinig ang 22 mag-aaral. Sinundan ito ng 20 na walang kumpiyansa sa sarili sa pagsasalita o pagbabalita sa harap ng madla. Samantala 19 naman ang nahihirapan kung paano matutunan ang kasanayan sa pagbabalita. Sumunod naman ang 15 ang nahihirapan sa pagbigkas ng mga salita sa pagbabalita. Kinakitaan naman ang $13 \mathrm{ng}$ kawalan ng interes sa gawaing pagbabalita. Ang 12 naman ay nahihirapang pumili mga salita upang maging matatas sa pagsasalita gamit ang wikang Filipino. Sinundan naman ng 11 na may kahirapan sa paglalahad ng mga lohikal, malinaw at organisadong mga detalye ng balita.

Nagpapakita lamang ito na malaki ang epekto sa kakayahang lingguwistik at diskorsal ng mga mag-aaral. Ang mga epektong ito ay naging sanhi upang hindi nila maipakita ang kanilang kasanayan.

\section{Talahanayan 2B}

Epekto ng Modular Distance Learning sa Kakayahang Diskorsal

\begin{tabular}{lcc}
\hline \multicolumn{1}{c}{ Epekto ng Modular Distance Learning sa Kakayahang Diskorsal } & f & Ranggo \\
\hline Kahirapan sa paglalahad ng mga lohikal, malinaw at organisadong mga detalye ng balita. & 11 & 7 \\
Nahihirapang pumili mga salita upang maging matatas sa pagsasalita gamit ang wikang Filipino. & 12 & 6 \\
Walang kumpiyansa sa sarili sa pagsasalita o pagbabalita sa harap ng madla. & 20 & 2 \\
Nahihirapan sa paglalapat nang angkop na lakas ng boses o tinig. & 22 & 1 \\
Nahihirapan sa pagbigkas ng mga salita sa pagbabalita. & 15 & 4 \\
Nahihirapan kung paano matutunan ang kasanayan sa pagbabalita. & 19 & 3 \\
Kawalan ng interes sa gawaing pagbabalita. & 13 & 5 \\
\hline
\end{tabular}

Ayon nga sa DepEd Order No. 12, s. 2020, ang integrasyon ng awtput sa "Basic Education- Learning Continuity Plan”, ang tanggapan ng Kalihim ay nagpalabas ng sumusunod na prinsipyo: una, protekahan ang kalusugan at kaligtasan ng bawat mag-aaral at lahat ng kawani ng pamahalaan at pagsugpo sa pagkalat ng Covid-19. Ikalawa, sikapin ang pagpapalaganap ng sulong edukalidad ng K-12 Kurikulum, katugmaan ng mga kagamitang panturo ayon sa mga mahahalagang kasanayang pampagkatuto, pagsasanay ng mga guro at mga mag-aaral at oryentasyon sa mga magulang o tagapangalaga ng bawat mag-aaral.

\section{Talahanayan 3}

Suliraning Kinakaharap ng mga Mag-aaral

\begin{tabular}{lcc}
\hline Suliraning Kinakaharap ng mga Mag-aaral & $\mathrm{f}$ & Ranggo \\
\hline Kakulangan sa materyal para sa mga mag-aaral. & 22 & 1 \\
Nahihirapan sa pag-unawa ang mga mag-aaral sa mga gawain sa gawaing pagkatuto. & 18 & 4.5 \\
Kakulangan ng angkop na kasanayan at kakayahan ng mag-aaral sa mga gawain. & 19 & 2 \\
Madaling masira ang mga gawain sa pagkatuto. & 15 & 6 \\
& 18 & 4.5 \\
Malabo at malilit na pagkakalimbag ng mga gawain sa pagkatuto. & 20 & 3 \\
Kakulangan sa mga sanggunian na magiging lunsaran ng pag-aaral & 12 & 7 \\
Kakulangan sa oras sa pagsagot sa gawaing pagkatuto. & & \\
\hline
\end{tabular}

Sa bahaging ito tinalakay ng mga guro at mag-aaral ang suliraning kinaharap sa Modular Distance Learning sa asignaturang Special Program on Journalism (Radio Broadcasting):

Makikita sa Talahanayan 3 ang mga Suliraning Kinakaharap ng mga Mag-aaral sa asignaturang Journalism ng Baitang 10- sa Special Program on Journalism (Radio Broadcasting). Nangunguna ang kakulangan sa materyal para sa mga mag-aaral na 22 kalahok. Nabanggit naman ng 19 na may kakulangan ng angkop na kasanayan at kakayahan ng mag-aaral sa mga gawain. Binanggit naman ng 20 na kakulangan sa mga sanggunian na magiging lunsaran ng pag-aaral. Ayon naman sa 18 nahihirapan sa pag-unawa ang mga mag-aaral sa mga gawain sa gawaing pagkatuto at malabo at malilit na pagkakalimbag ng mga gawain sa pagkatuto. Sinabi naman ng 15 na madaling masira ang mga gawain sa pagkatuto. Samantala ayon sa 12 may kakulangan sa oras sa pagsagot sa gawaing pagkatuto.

Sinuportahan naman ito mula sa panayam sa mga Guro 
Pante, D. J. B.

Ayon kay Guro A:

"Sa modular distance learning ang mga mag-aaral ay hindi umuunawa sa binabasa at hindi sumusunod sa partikular na na Panutol direksyon. Kawalan ng interes sa pagbabasa ng mga artikulo. Hindi pagsasagawa ng mga gawain. Hindi maalam sa wastong pagbabaybay at wastong balarila”

Sinabi ni Guro B:

"Hindi wastong balarila ng sagot ng mga mag-aaral sa gawaing pagkatuto. Walang kasiguraduhan kung ang kanilang mga sagot ay sariling gawa. Hindi malinang nang maayos ang kasanayan sa diskorsal ng mga mag-aaral dahil sa modular ang pamamaraang ng pagtuturo. Mahirap matukoy ang kalakasan at kahinaan ng kasanayan ng mga mag-aaral.

Batay naman kay Guro C:

"Naging suliranin ko ang kakulangan sa mga kagamitan ng mag-aaral at mga gadget na magagamit sa pag-aaral, komunikasyon at kawalan ng koneksyon sa internet”

Nabanggit naman ni Guro D:

\begin{abstract}
“Zero ang materyal sa pagtuturo dahil walang dumating mula sa DepEd Central Office kaya't gumawa ng sariling materyal na naaayon sa Most Essential Learning Competencies. Mahirap magturo ng kasanayan sa pagsulat dahil hindi mo sila nakikita at personal na namomonitor at sa tests lang nakasalalay. Hindi ka sure sa authencity at originality ng work (Kahit di sya plagiarized di mo alam kung siya talaga gumawa o pinagawa nya sa iba. Ang bilis makalimot ng lesson ng mga bata (walang retention eversince pero mas malala ngayon... after gawin ang module, wala na din sa isip nila). Di nadedevelop ng mabuti expected skills ng bata at di sila exposed sa activities na makakadevelop sa kanilang full potential. (Kung ang mga students natin hindi financially challenged at may stable signal sa atin, kaya naman sana itaguyod ang distance learning kasi before pandemic may distance learning, particularly online learning na, kaso di lang exposed ang karamihan).
\end{abstract}

Sa Talahanayan 4 Makikita ang Mungkahing Gawain sa Paglinang ng Kakayahang Lingguwistik at Diskorsal ng mga mag-aaral sa asignaturang Journalism Baitang 10- sa Special Program on Journalism (Radio Broadcasting). Pagbuo ng karagdagang materyal o gawain sa pagkatuto para sa mag-aaral at pagbuo ng karagdagang materyal o gawain sa pagkatuto para sa mag-aaral 4 ang nagbigay pahayag ukol dito. Ayon naman sa 3 p agdalo ng mga guro at mag-aaral sa mga webinar/traning na may kaugnayan sa Radio Broadcasting at karagdagang mga video clips ng pagbabalita. Samantala 5 naman ang nagsabi ng karagdagang “drill” sa gawaing pagsasalita o pagbabalita.

\title{
Talahanayan 4
}

Mungkahing Gawain sa Paglinang ng Kakayahang Lingguwistik

\begin{tabular}{|c|c|c|}
\hline $\begin{array}{c}\text { Mungkahing Gawain sa Paglinang ng Kakayahang Lingguwistik at Diskorsal ng mga } \\
\text { mag-aaral }\end{array}$ & $\mathrm{f}$ & Ranggo \\
\hline Karagdagang "drill" sa gawaing pagsasalita o pagbabalita & 2 & 5 \\
\hline Pagbuo ng karagdagang materyal o gawain sa pagkatuto para sa mag-aaral. & 4 & 1.5 \\
\hline $\begin{array}{l}\text { Pagdalo ng mga guro at mag-aaral sa mga webinar/traning na may kaugnayan sa Radio } \\
\text { Broadcasting }\end{array}$ & 3 & 3.5 \\
\hline Karagdagang mga video clips ng pagbabalita. & 3 & 3.5 \\
\hline $\begin{array}{l}\text { Magkaroon ng Blended Learning para sa Performans ng mag-aaral sa pagbabalita sa } \\
\text { audio-visual room(Observe Health Protocol) }\end{array}$ & 4 & 1.5 \\
\hline
\end{tabular}

Pinagtibay ito ng pahayag ni Llego (2020), ang "Distance learning Modalities" ay maaaring makatulong sa pagpapa-unlad ng access sa edukasyon, at paglinang sa pagkatuto, maging sa pagpapalaganap ng

6 Consortia Academia Publishing (A partner of Network of Professional Researchers and Educators) 
Kakayahang lingguwistik at diskorsal ng baitang 10 - special program on journalism: Isang pagsusuri

“Basic-Education- Learning Continuity Plan" sa pagtugon sa hamon ng pandemya tulad ng ng Covid-19, pagkalat ng nakahahawang sakit tulad ng Ebola, at mga natural na kalamidad. Sa pamamagitan ng pagsunod sa apat na pamamaraan ng "distance learning modalities" maaaring maging susi ito upang maitawid ang pagtuturo at pagkatuto.

\section{Konklusyon}

Ang lebel ng kakayahang lingguwistik ng mga mag- aaral sa asignaturang Journalism ng Baitang 10- sa Special Program on Journalism (Radio Broadcasting) ay 82.39\% at kakayahang diskorsal ay 83. $31 \%$ ito ay nagpapakita na sila ay nasa katamtaman lebel ng kasanayan. May epekto ng Modular Distance Learning sa kakayahan ng mga mag-aaral sa asignaturang Journalism Baitang 10- Special Program on Journalism (Radio Broadcasting). May mga suliraning kinakaharap ng mga mag-aaral sa Modular Distance Learning sa asignaturang Journalism ng Baitang 10- Special Program on Journalism (Radio Broadcasting). May mga mungkahing gawain ang maaaring gawin upang mas higit na malinang ang kakayahang lingguwistik at diskorsal ng mga mag-aaral sa asignaturang Journalism Baitang 10-Special Program on Journalism (Radio Broadcasting).

\subsection{Rekomendasyon}

Inirerekomenda na magsagawa pa ng mga karagdagang mga materyal na makatutulong sa paglinang ng kakayahang lingguwistik na kung saan ang pokus ay wastong balarila sa pagsulat ng mga mag-aaral. Gayundin paglinang sa kakayahang diskorsal ng mga mag-aaral na ang pokus ang pamamaraan pagsasalita o pagpapahayag. Magsagawa ng Blended Learning na kung saan naglalayong magkaroon ng mas malawakang paglinang sa lebel ng performans ng mga mag-aaral sa kakayahang lingguwistik at diskorsal. Paggamit ng mga guro ng karagdagang video clips at pagsasagawa ng mga drill na lilinang sa kakayahan ng mag-aaral. Paglahok ng mga guro at mag-aaral sa webinar/training na may worksyap upang mas mahikayat ang mga mag-aaral sa pagganap. Mabigyang pansin ng admisnistrasyon para sa pagkakaroon ng mga karagdagang kagamitan/gadget para sa isinasagawang radio broadcast.

\section{Sanggunian}

Badayos, P. B. (2008). Metodolohiya sa Pagkatuto ng/sa Filipino. Mga Teorya, Simulain at Estratehiya. Mutya Publishing.

Cabigao, J. R. (2021). School-Based Assessment Framework Version 2.0 (The New Normal). IJAMR, 5, 106-108.

Chomsky, N. (1965). Retrieved from https://www.elcomblus.com/kakayahang-lingguwistiko/

Department of Education. (2020). Retrieved from https://www.deped.gov.ph/wp-content/uploads/2015/04/DO_s2015_08.pdf

Konstitusyong ng Republika ng Pilipinas. (1991). Retrieved from https://www.officialgazette.gov.ph/downloads/1987/02feb/19870211-Konstitusyon-CCA.pdf

Llego, M. A. (n.d). DepEd Learning Delivery Modalities for School Year 2020-2021. TeacherPh. Retrieved from https://www.teacherph.com/deped-learning-delivery-modalities/

United Nations Children's Fund. (2020). Retrieved from https://www.unicef.org/media/100946/file/UNICEF\%20Annual\%20Report\%202020.pdf

United States Department of Education, Office of Civil Rights, \& Office of Special Education and Rehabilitative Services. (2020). Supplemental Fact Sheet Addressing the Risk of Covid-19 in Preschool, Elementary, Secondary Schools While Serving Children with Disabilities. Retrieved from https://www2.ed.gov/about/offices/list/ocr/frontpage/faq/rr/policyguidance/Supple\%20\%Fact\%20Sheet $\% 203.21 .20 \% 20$ FINAL.pdf. 
Pante, D. J. B. 